\title{
Positron confinement in embedded lithium nanoclusters
}

\author{
M. A. van Huis, A. van Veen, H. Schut, C. V. Falub, S. W. H. Eijt, and P. E. Mijnarends \\ Interfaculty Reactor Institute, Delft University of Technology, Mekelweg 15, NL-2629 JB Delft, The Netherlands \\ J. Kuriplach \\ Department of Low Temperature Physics, Charles University, V Holešovičkách 2, CZ-180 00 Prague, Czech Republic
}

(Received 2 May 2001; revised manuscript received 4 October 2001; published 8 February 2002)

\begin{abstract}
Quantum confinement of positrons in nanoclusters offers the opportunity to obtain detailed information on the electronic structure of nanoclusters by application of positron annihilation spectroscopy techniques. In this work, positron confinement is investigated in lithium nanoclusters embedded in monocrystalline $\mathrm{MgO}$. These nanoclusters were created by means of ion implantation and subsequent annealing. It was found from the results of Doppler broadening positron beam analysis that approximately $92 \%$ of the implanted positrons annihilate in lithium nanoclusters rather than in the embedding $\mathrm{MgO}$, while the local fraction of lithium at the implantation depth is only 1.3 at. \%. The results of two-dimensional angular correlation of annihilation radiation confirm the presence of crystalline bulk lithium. The confinement of positrons is ascribed to the difference in positron affinity between lithium and $\mathrm{MgO}$. The nanocluster acts as a potential well for positrons, where the depth of the potential well is equal to the difference in the positron affinities of lithium and $\mathrm{MgO}$. These affinities were calculated using the linear muffin-tin orbital atomic sphere approximation method. This yields a positronic potential step at the $\mathrm{MgO} \| \mathrm{Li}$ interface of $1.8 \mathrm{eV}$ using the generalized gradient approximation and $2.8 \mathrm{eV}$ using the insulator model.
\end{abstract}

DOI: 10.1103/PhysRevB.65.085416

PACS number(s): 61.46.+w, 71.60.+z, 78.70.Bj

\section{INTRODUCTION}

Metal and semiconductor nanoclusters are studied extensively because of their linear and nonlinear optical properties $^{1-3}$ and their electronic features as quantum dots. ${ }^{4-6}$ The optical and electronic properties are strongly dependent on the nanocluster size, which is due to quantum size effects such as the confinement of surface plasmons and the discretization of energy levels below a certain cluster size. The engineering of quantum dots offers great opportunities for the future as the material properties, such as the band gap and melting temperature of semiconductor clusters, can be tuned by varying the cluster size. ${ }^{7}$

Positron confinement is a newly investigated type of quantum confinement in nanoclusters and can serve as a powerful tool to investigate the electronic structure of nanoclusters. Positrons are known as sensitive self-seeking probes of defects in materials and can provide detailed information on the electronic structure of defects or bulk materials ${ }^{8,9}$ such as the morphology of Fermi surfaces. ${ }^{10,11}$ The concept of positron confinement was introduced by Nagai et al. ${ }^{12}$ Under certain conditions, nanoclusters act as a potential well to positrons; i.e., the wave function of the positron is spatially confined within the nanocluster in three dimensions. A positron confined in a nanocluster will annihilate there and will thus provide unique information on the electronic and crystal structure of the nanocluster, which can be quite different from the bulk material of the atoms constituting the nanocluster. Previously, positron confinement in $\mathrm{Cu}$ nanoclusters embedded in Fe was investigated. ${ }^{12}$ By analysis of the $S$ and $W$ parameters of the Doppler broadening curves these authors found that, after thermal aging, approximately $90 \%$ of all positrons annihilate in the $\mathrm{Cu}$ nanoclusters while $\mathrm{Cu}$ makes up only $1.0 \mathrm{wt} \%$ of the $\mathrm{Fe}: \mathrm{Cu}$ composite material.
The positron confinement was confirmed by coincidence Doppler broadening (CDB) measurements and was ascribed to a difference in positron affinity between $\mathrm{Cu}$ and $\mathrm{Fe}$ of 1.0 $\mathrm{eV}$. If the positron affinity of the cluster material is lower than the positron affinity of the host material, the cluster will act as a potential well to positrons, as will be explained in Sec. II.

In this work, positron confinement is investigated in $\mathrm{Li}$ nanoclusters embedded in monocrystalline $\mathrm{MgO}$. In order to study the material properties of nanoclusters, it can be useful to embed nanoclusters in stable and inert ceramics such as $\mathrm{MgO}$, which can be achieved by ion implantation and subsequent annealing. ${ }^{13}$ It is expected that the wide-band-gap material $\mathrm{MgO}(7.8 \mathrm{eV})$ does not interact with the electronic structure of metal and semiconductor clusters. ${ }^{14,7}$ It is also optically transparent in a large frequency band, which facilitates optical studies. Furthermore, the high melting point of $\mathrm{MgO}(3070 \mathrm{~K})$ allows a study of phase transitions of nanoclusters. The optical and structural properties of Li nanoclusters embedded in $\mathrm{MgO}$ have been investigated previously by photoabsorption spectroscopy, transmission electron microscopy, and electron diffraction. ${ }^{15-17}$ It was found that small Li clusters $(<20 \mathrm{~nm})$ adopt the unusual fcc crystal structure while large Li clusters $(>40 \mathrm{~nm})$ adopt the regular bcc crystal structure. This is a so-called extrinsic size effect: small Li clusters adopt a different phase in order to fit better in the $\mathrm{MgO}$ matrix. The lattice parameter of fcc lithium is closer to that of $\mathrm{MgO}$, which reduces the formation energy of the $\mathrm{MgO} \| \mathrm{Li}$ interface. The fcc phase for bulk $\mathrm{Li}$ is otherwise only observed at low temperatures and high pressures. ${ }^{18,19} \mathrm{~A}$ similar effect was reported in the work mentioned above ${ }^{12}$ where the crystal structure of $\mathrm{Cu}$ nanoclusters embedded in Fe is expected, on the basis of lattice parameter similarity, to be bcc rather than fcc. 
The nanoclusters investigated in this work were created by ion implantation and subsequent annealing. It was shown previously ${ }^{20}$ that Doppler broadening positron beam analysis (PBA) and two-dimensional angular correlation of annihilation radiation (2D-ACAR) measurements show a very high fraction of positron annihilations in Li nanoclusters, indicating possible positron quantum confinement. Below we will first discuss the concepts of positron affinity and the positronic potential well. After describing the experimental techniques and procedures, the results from positron annihilation techniques are presented and analyzed in order to obtain a value for the fraction of positrons annihilating in lithium nanoclusters. Furthermore, the positron affinities of $\mathrm{MgO}$ and fcc and bec lithium are calculated in order to determine the drop of the positronic potential at the $\mathrm{MgO} \| \mathrm{Li}$ interface. The confinement of positrons in $\mathrm{Li}$ nanoclusters is then discussed using the experimental and computational results.

\section{THEORETICAL METHODS AND CONCEPTS}

Annihilation of positrons with electrons in solids yields information on the momentum distribution of these electrons. The electronic momentum distribution is reflected in the Doppler broadening of the $511 \mathrm{keV}$ annihilation peak. Positron annihilation with low-momentum valence or conduction electrons results in a small Doppler shift, contributing to the center of the peak. Annihilation with highmomentum core electrons results in a large Doppler shift, contributing to the wings of the $511 \mathrm{keV}$ annihilation peak. The shape of the $511 \mathrm{keV}$ peak is characterized by the socalled $S$ and $W$ parameters, ${ }^{21,22}$ as defined in Fig. 1. Here the $S$ parameter indicates the relative contribution from valence and conduction electrons while the $W$ parameter represents the relative contribution from core electrons. When combined with a slow positron beam, this method is called Doppler broadening positron beam analysis. The $S$ and $W$ parameters can be considered as a specific bulk property for every material. In the case of composite materials, e.g., metallic nanoclusters embedded in insulating oxides, the overall annihilation distribution can be modeled as a linear combination of the annihilation distribution of each of the composing materials. The $S$ parameter of the composite material then equals

$$
S_{\text {comp }}=f_{\text {cluster }} S_{\text {cluster }}+f_{\text {host }} S_{\text {host }} \text {, }
$$

where $S_{\text {cluster }}$ and $S_{\text {host }}$ are the bulk $S$ parameters of the nanoclusters and the embedding material, respectively. $f_{\text {cluster }}$ and $f_{\text {host }}$ are the fractions of annihilation in the clusters and in the embedding host material $\left(f_{\text {cluster }}+f_{\text {host }}=1\right)$. Here it is assumed that the composite material is defect free and that positrons do not annihilate at the cluster-host interface. Of course, the principle of superposition of annihilation distribution can be applied to other positron annihilation methods as well, including coincidence Doppler broadening and twodimensional angular correlation of annihilation radiation. Equation (1) applies to a "bulk" composite material. In the case of, e.g., an ion-implanted layer investigated using a pos-

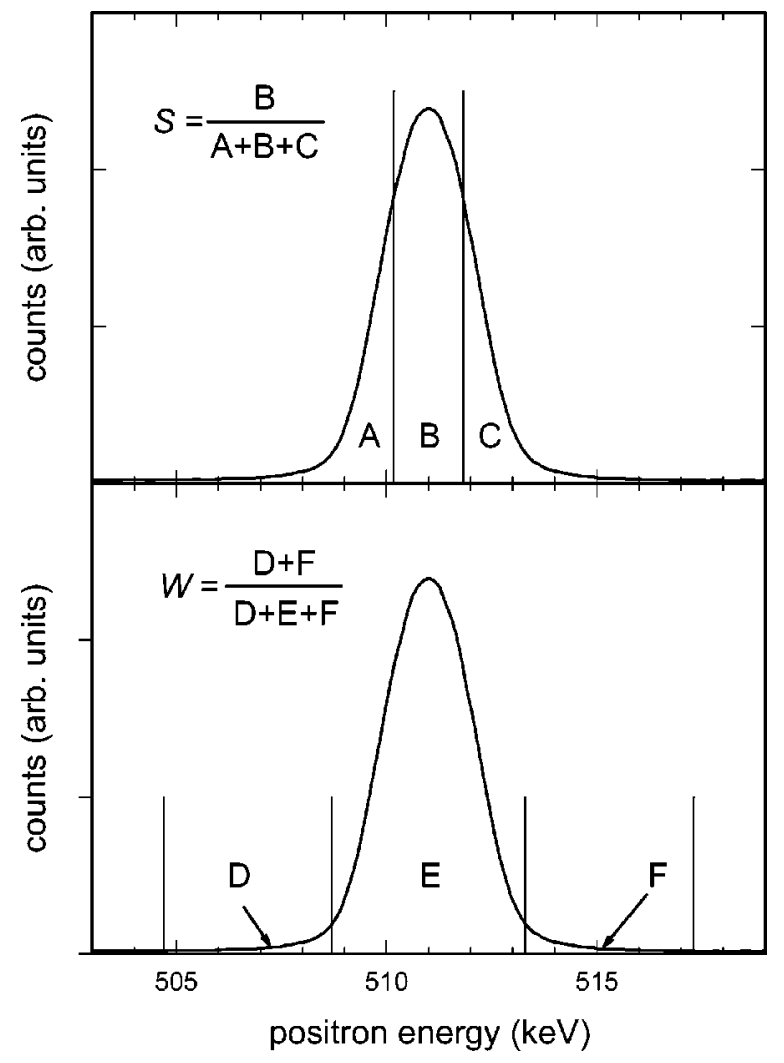

FIG. 1. Definition of the $S$ and $W$ parameter with the corresponding energy windows used in the analysis of the $511 \mathrm{keV}$ positron annihilation peak.

itron beam, the $S$ parameter obtained at a certain positron implantation energy consists of contributions from the surface and various layers,

$$
S(E)=f_{\text {surf }}(E) S_{\text {surf }}+f_{1}(E) S_{1}+f_{2}(E) S_{2}+\cdots+f_{n}(E) S_{n} .
$$

Here the material consists of $n$ layers and the distribution of the fractions $\left(f_{\text {surf }}+f_{1}+\cdots+f_{n}=1\right)$ depends on the positron energy. Please note that Eq. (1) refers to defect (or material) fractions while Eq. (2) refers to layer fractions. Formulas analogous to Eqs. (1) and (2) are valid for the $W$ parameter. Fitting methods such as VEPFIT (Ref. 23) and SWAN (Ref. 24) exist, which yield accurate values for the $S$ and $W$ parameters of the various layers and the fractions of positrons annihilating in these layers as a function of positron implantation energy. This is achieved by solving the time-averaged diffusion equation. ${ }^{23}$ Once the $S$ parameter of a certain layer has been found using the VEPFIT code, Eq. (1) can be used in order to analyze further the defect fractions within that layer.

Assuming that the nanoclusters are homogeneously distributed, the annihilation fractions $f_{\text {cluster }}$ and $f_{\text {host }}$ are determined by the diffusion length of the positrons, the concentration of clusters, and the difference in positron affinity between both materials. Although the overall concentration of atoms constituting the clusters in the host material is usually small, the annihilation fraction in the clusters can be 


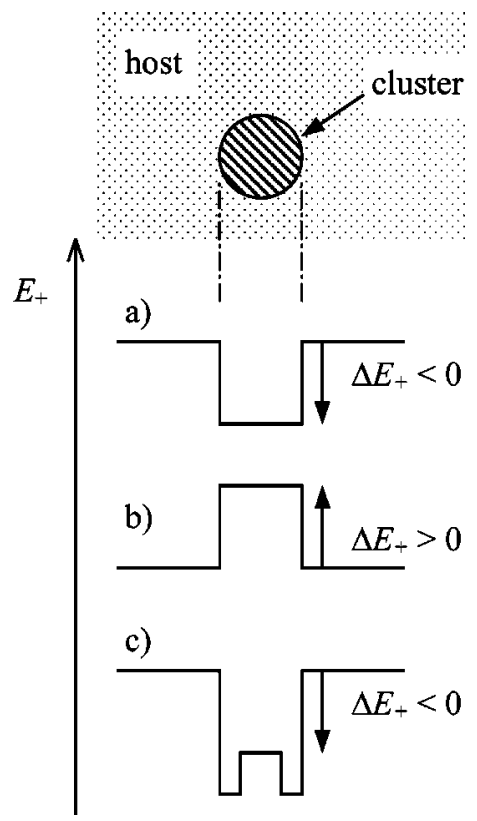

FIG. 2. Diagram of the positron potential energy as a function of the distance from the center of the nanocluster. (a) Nanocluster acts as a positronic potential well with depth $\Delta E_{+}$. (b) Nanocluster acts as a potential barrier with step size $\Delta E_{+}$. (c) Positron trapping at defects at the cluster-host interface.

high with respect to the annihilation fraction in the host material when certain conditions with respect to the positron affinities are met.

Since the Fermi levels of the materials in contact become equal, ${ }^{25}$ the positron affinity is defined as ${ }^{26}$

$$
A_{+}=\mu_{-}+\mu_{+}=-\left(\phi_{-}+\phi_{+}\right),
$$

where $\mu_{-}$and $\mu_{+}$are the electron and positron chemical potentials, and $\phi_{-}$and $\phi_{+}$the electron and positron work functions, respectively. Values for the positron affinity are commonly expressed in $\mathrm{eV}$ and are negative for most materials. At the interface between two materials, positrons will encounter a potential step that is equal to the difference in the positron affinities of both materials, ${ }^{26}$

$$
\Delta E_{+}^{A B}=A_{+}^{A}-A_{+}^{B} .
$$

A necessary condition for positron confinement in a cluster (A) embedded in a host $(B)$ is $\Delta E_{+}^{A B}<0$. Then the cluster represents a potential well for positrons. However, there must be at least one bound state in such a well, which determines the minimum size of the cluster. Assuming a spherical potential well this condition reads ${ }^{26}$

$$
R_{\text {min }}>\frac{\pi \hbar}{\sqrt{8 m_{0} \Delta E_{+}^{A B}}},
$$

where $R$ is the radius of the cluster and $m_{0}$ is the positron mass. In Fig. 2, several possibilities for the positron potential energy as a function of the distance from the center of the nanocluster are plotted schematically. In Fig. 2(a), $\Delta E_{+}^{A B}$ $<0$. Here the nanocluster acts as a positronic potential well with depth $\Delta E_{+}^{A B}$. Positrons can be confined in this nanocluster provided that condition (5) is satisfied. In Fig. 2(b), $\Delta E_{+}^{A B}>0$. The nanocluster acts as a three-dimensional potential barrier and cannot confine positrons. Figure 2(c) shows a situation where the positron affinity of the nanocluster material is lower than the positron affinity of the host material, but positron confinement is unlikely as the positron is trapped at the interface. Often there is some lattice mismatch due to the different crystal structures of the cluster and the embedding matrix. At the interface, this may result in structural and electronic defects, which can act as a trap for positrons. In the case of, e.g., a $\mathrm{Si} \| \mathrm{SiO}_{2}$ interface, there is evidence of preferential trapping at this interface. ${ }^{27}$

Usually, the positron affinity is determined experimentally by measuring the electron and positron work functions ${ }^{28}$ [see Eq. (3)]. Alternatively, the positron affinity can be derived from the positronium (Ps) formation potential $\varepsilon_{P s}$, which can be determined experimentally as the negative of the maximum kinetic energy of positronium atoms ejected from the sample into vacuum. The positronium formation potential is related to the positron affinity as ${ }^{26}$

$$
\varepsilon_{P s}=\phi_{+}+\phi_{-}-6.8 \mathrm{eV}=-A_{+}-6.8 \mathrm{eV},
$$

where $6.8 \mathrm{eV}$ is the binding energy of the positron-electron pair constituting a positronium atom in vacuum. Two experimental time-of-flight studies of $\mathrm{MgO}$ (Refs. 29 and 30) report positronium emission peak energies of 4.5 and $2.6 \mathrm{eV}$, respectively. Applying Eq. (6) then yields values for the positron affinity for $\mathrm{MgO}$ of -2.3 and $-4.2 \mathrm{eV}$, respectively. Apart from the quantitative discrepancy, the interpretation of these results is complicated by the fact that the emission energy spectra are quite broad. Furthermore, the unknown binding energy of Ps at the $\mathrm{MgO}$ surface ( $\mathrm{MgO}$ is too dense a material for Ps to be formed in the bulk) and the uncertainty about whether valence or conduction electrons participate in Ps formation obscure a direct translation to the positron affinity for $\mathrm{MgO} .^{29,30}$ Summarizing, determining positronium formation potentials at the surface of insulating oxides such as $\mathrm{MgO}$ is experimentally difficult, which results in the different values reported in the literature.

Therefore, we turn to $a b$ initio calculations to determine the positron affinities. As the calculation of electron and positron work functions requires a careful treatment of the material's surface, we prefer to determine $A_{+}$through the electron and positron chemical potentials [see Eq. (3)], the calculation of which is a standard procedure in "bulk" electronic structure computational methods. It should be noted that $A_{+}$is a bulk characteristic of a material (see Refs. 9 and 26 for details). For the calculation of the positron affinities and lifetimes in the materials studied, ${ }^{9}$ the linear-muffin-tinorbital (LMTO) method was used within the atomic sphere approximation (ASA). ${ }^{31,32}$ Within the framework of this method the crystal is divided into slightly overlapping spheres (called atomic spheres) centered at the positions of the nuclei. The sum of the volumes of the spheres is equal to the volume of the crystal. However, the rocksalt structure of $\mathrm{MgO}$ is not as closely packed as the fcc and bec structures considered for $\mathrm{Li}^{16}$ Therefore we had to introduce empty 
TABLE I. Structural data.

\begin{tabular}{lclc}
\hline \hline & Structure & $a_{0}(\AA)$ & at. volume $\left(\AA^{3}\right)$ \\
\hline $\mathrm{MgO}$ & rocksalt & $4.212^{\mathrm{a}}$ & - \\
$\mathrm{Li}$ & fcc & $4.40^{\mathrm{b}}$ & 21.3 \\
$\mathrm{Li}$ & $\mathrm{bcc}$ & $3.51^{\mathrm{b}}$ & 21.6 \\
\hline \hline
\end{tabular}

${ }^{\mathrm{a}}$ Ref. 52.

${ }^{b}$ Ref. 16.

spheres $^{31}$ into the $\mathrm{MgO}$ lattice to describe properly the distribution of the electron and positron densities and potentials in the interstitial region. We considered the case where the empty spheres occupied $30 \%$ of the cell volume. In the following this case is referred to as $\mathrm{MgO} \mathrm{II}$, in contrast to $\mathrm{MgO}$ I where no empty spheres were included for comparison. Choosing the $30 \%$ filling with empty spheres leads to a reasonable overlap between neighboring $\mathrm{Mg}-\mathrm{O}$ spheres in the MgO lattice. ${ }^{31}$ The lattice constants $a_{0}$ used in our calculations are collected in Table I. As for the positron calculations, in $\mathrm{Li}$ we used the parametrizations of the positron correlation potential and enhancement factor given by Boroński and Nieminen $^{33}$ within the framework of the local density approximation (LDA). The corresponding results will further be denoted as LDA, whereas the results obtained using the generalized gradient approximation ${ }^{34}$ will be marked GGA. In the case of $\mathrm{MgO}$ we also employed the GGA, but instead of the LDA we made use of the so-called semiconductor (SM) and insulator (IM) models, ${ }^{35}$ which take into account incomplete positron screening in nonmetallic systems (i.e., systems having a band gap; $\mathrm{MgO}$ is an insulator). A highfrequency dielectric constant $\varepsilon_{\infty}=3.0$ was employed in the SM and IM calculations. This constant was derived from the optical constants of $\mathrm{MgO}$ (Ref. 36) in the visible spectrum $(n, k)=(1.73,0.00)$ using the relationship $\varepsilon=n^{2}-k^{2}$. Finally, the exchange-correlation potential for electrons parametrized according to Von Barth and $\mathrm{Hedin}^{37}$ was used in the calculations of the electronic structure of $\mathrm{MgO}$ and $\mathrm{Li}$.

For completeness, the positron lifetime $(\tau)$ in defect-free $\mathrm{MgO}$ and $\mathrm{Li}$ was calculated employing the well-known formula ${ }^{9}$

$$
1 / \tau=\pi r_{0}^{2} c \int n_{-}(\mathbf{r}) n_{+}(\mathbf{r}) \gamma\left[n_{-}(\mathbf{r})\right] \mathrm{d} \mathbf{r} .
$$

Here, $n_{-}$and $n_{+}$denote the electron and positron density, respectively, and $\gamma$ stands for the enhancement factor describing the pileup of electrons around a positron $\left(r_{0}\right.$ is the classical radius of the electron and $c$ the speed of light). The form of $\gamma$ within the LDA, GGA, SM, and IM approaches was used in the corresponding calculations. Other computational details are described in Ref. 38.

\section{EXPERIMENT}

Epi-polished monocrystals of $\mathrm{MgO}(100)$ of size $10 \times 10$ $\times 1 \mathrm{~mm}^{3}$ were implanted with $1.0 \times 10^{16} \mathrm{~cm}^{-2}{ }^{6} \mathrm{Li}$ ions at an energy of $30 \mathrm{keV}$. After the implantation the crystals were annealed in air in steps from room temperature to $1200 \mathrm{~K}$.
The annealing temperatures were 550, 750, 950, 1100, and $1200 \mathrm{~K}$ for periods of $30 \mathrm{~min}$. Photoabsorption spectroscopy was used to detect Mie plasmon resonance due to the presence of lithium nanoclusters. Furthermore, the neutron depth profiling (NDP) technique, using the nuclear reaction ${ }^{6} \mathrm{Li}(\mathrm{n}, \alpha){ }^{3} \mathrm{H}$, was applied to determine the depth profile of ${ }^{6} \mathrm{Li}$ after the annealing steps. The results of these two methods have already been discussed in an earlier paper, which focused on the defect evolution during the annealing procedure. ${ }^{20}$ In the present work we address the confinement of positrons in lithium nanoclusters, and therefore only the positron experimental techniques applied to the samples are presented in this paper.

After ion implantation and after each annealing step, the samples were analyzed with Doppler broadening positron beam analysis using a monoenergetic $0-30 \mathrm{keV}$ positron beam. The energy resolution of the PBA setup is $1.2 \mathrm{keV}$. After the $950 \mathrm{~K}$ annealing step, one of the samples was also analyzed with 2D-ACAR. The 2D-ACAR setup of the Anger-camera type is coupled to a high-intensity positron beam with a flux of $8 \times 10^{7} e^{+} \mathrm{s}^{-1}$, allowing depth-selective $2 \mathrm{D}-\mathrm{ACAR}^{39}$ The angular resolution of this system is 1.1 $\times 1.4 \mathrm{mrad}^{2}$. The sample measured with $2 \mathrm{D}$-ACAR was identical to the other samples (which was ascertained by means of photoabsorption spectroscopy and PBA), apart from a 15-nm-thick layer of Al deposited on the sample surface after the $950 \mathrm{~K}$ annealing step to prevent buildup of electric charge. The 2D-ACAR distribution was collected at a positron implantation energy of $4 \mathrm{keV}$, which corresponds to a mean positron implantation depth at the center of the layer containing the lithium nanoclusters. This energy was chosen on the basis of depth profiling performed on the same sample by means of Doppler broadening PBA. Furthermore, an $\mathrm{MgO}$ bulk 2D-ACAR distribution was collected for reference purposes from an as-received sample of $\mathrm{MgO}(100)$. The use of a conventional ${ }^{22} \mathrm{Na}$ source in this latter measurement renders any surface effects negligible.

\section{RESULTS}

\section{A. Positron beam analysis}

Figure 3 shows the $S$ and $W$ parameters as a function of positron implantation energy after lithium ion implantation and after annealing at the indicated temperatures. The solid lines represent the result of simulations performed with the VEPFIT code, as will be discussed below. The $S$ parameter shows a spectacular increase in the lithium ion implantation range (corresponding to a positron energy of 3-4 keV) after annealing at temperatures of $750 \mathrm{~K}$ and $950 \mathrm{~K}$. At higher annealing temperatures the $S$ parameter in the ion implantation layer drops considerably and after annealing at $1200 \mathrm{~K}$ the peak almost disappears in this range. At larger depths (corresponding to positron implantation energies of 7-15 $\mathrm{keV}$ in Fig. 3), the $S$ parameter drops below the bulk value of $\mathrm{MgO}$ (i.e., 0.468) after annealing at $1200 \mathrm{~K}$. Such a drop of the $S$ parameter below the bulk value of $\mathrm{MgO}$ as a result of ion implantation and subsequent annealing has not been ob- 


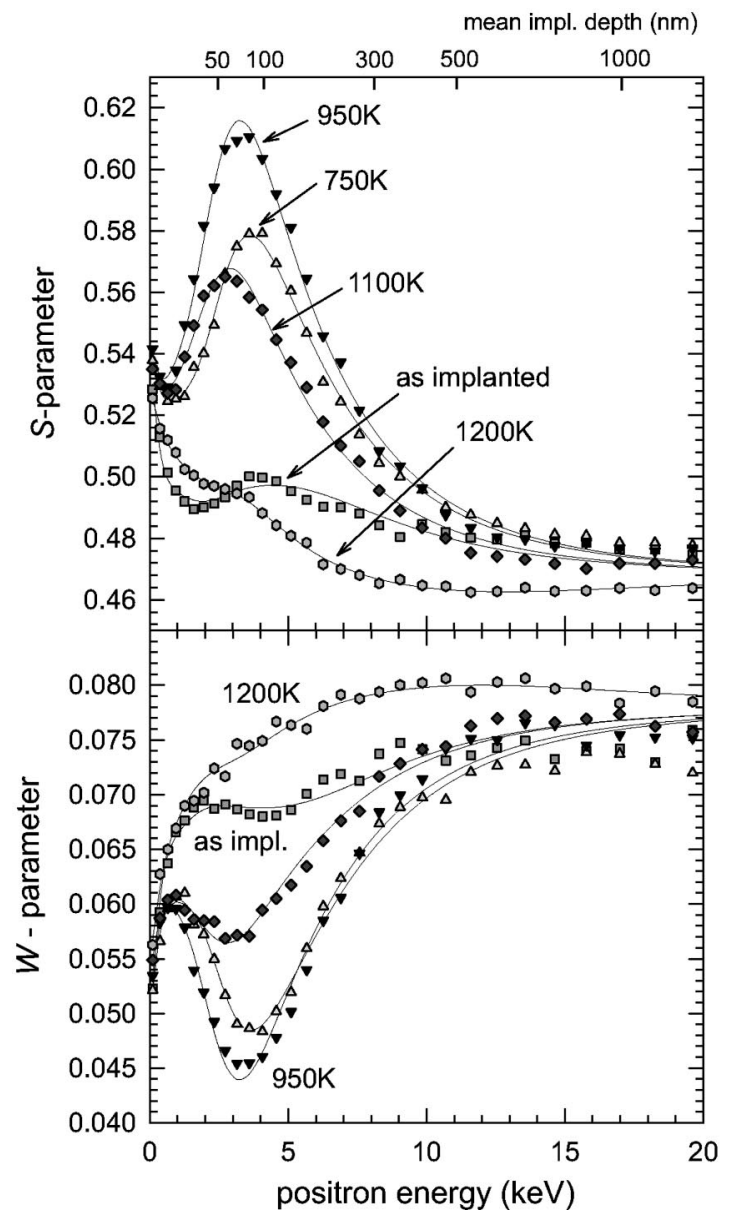

FIG. 3. $S$ and $W$ parameters as a function of positron energy. The solid lines are the result of VEPFIT modeling.

served before and we attribute this effect to positron annihilation in small Li-related defects, as will be discussed in more detail below.

The experimental $S$ and $W$ parameter curves shown in Fig. 3 have been fitted using the VEPFIT $\operatorname{code}^{23}$ in order to gain more insight into defect evolution and in order to derive the contributions of the various layers to the annihilation distribution. A four-layer model was used. The principal defect types and the corresponding diffusion lengths that were used as input to the VEPFIT code are listed in Table II. The choice for this model is mainly based on defect analysis performed on previously ion-implanted $\mathrm{MgO}$ samples that were analyzed with techniques such as transmission electron microscopy, positron annihilation spectroscopy, and neutron depth profiling. ${ }^{20,40,41}$ In particular, there is a subsurface layer con- taining the ion implanted species and the main implantation defects (layer 2). Dislocation loops are formed at smaller depths in the $\mathrm{MgO}$ top layer (layer 1) and below the ion implantation layer. ${ }^{40}$ The presence of dislocation loops in $\mathrm{MgO}$ does not affect the $S$ parameter significantly, but it does shorten the diffusion length compared to the bulk values. In the layer below the ion implantation layer (layer 3) there are not only dislocation loops but also a tail of implanted species as observed by $\mathrm{NDP}^{20}{ }^{20}$ possibly because of channeling effects. It was estimated that about $6 \%$ of the implanted species end up in this range. Due to the low atomic concentration of $\mathrm{Li}$ in this layer, we expect small $\mathrm{Li}$-related defects rather than metallic Li clusters (see the discussion on the defect evolution of layer 3 in Fig. 3 below). The fourth layer is the $\mathrm{MgO}$ bulk. In order to monitor the defect evolution, the $S$ and $W$ parameters of layer 2 (ion implantation layer) and layer 3 (ion implantation tail) were fitted. The layer boundaries determining the position of the ion implantation layer have been allowed to relax as the $S$ parameter peak shifts to somewhat lower positron energies when the annealing temperature increases (compare, for example, the $750 \mathrm{~K}$ and $1100 \mathrm{~K} S$ parameter curves in Fig. 3). This might be due to a recombination of implanted lithium atoms and lithium clusters with vacancies and small vacancy clusters created by ion implantation. The main ion implantation damage is always located at slightly smaller depths than the range of the implanted ions.

The fitted curves are depicted as solid lines in Fig. 3. The agreement with the experimental results is reasonable, considering the fact that the same model has been applied to all data sets. In the case of the $S$ and $W$ graphs corresponding to the $1200 \mathrm{~K}$ annealing step the $\mathrm{MgO}$ top layer was omitted since it was not possible to distinguish between the ion implanted layer and the $\mathrm{MgO}$ top layer. The fitted results yield boundaries $d_{1}$ and $d_{2}$ of the ion implanted layer of approximately 45 and $115 \mathrm{~nm}$, respectively, shifting to slightly lower values with increasing annealing temperature. The fitted values of $S$ and $W$ are presented in Fig. 4(b).

Figure 4 shows the $S$ parameter plotted versus the $W$ parameter. Every bulk material and every defect type has a characteristic $S$ and $W$ parameter. A few of these typical $S, W$ combinations are indicated in Fig. 4 with open circles and are called cluster points. The cluster points corresponding to pure $\mathrm{MgO}$ and bcc Li were determined experimentally from bulk samples of these materials. By plotting experimental or fitted $S, W$ values in an $S$-W plot with, e.g., the positron energy as the running parameter, the defect status at a certain depth in the material can be analyzed by means of comparison with the characteristic $S, W$ cluster points for defects and

TABLE II. VEPFIT model used to fit the $S$ and $W$ parameter curves shown in Fig. 3.

\begin{tabular}{lccccc}
\hline \hline & depth $(\mathrm{nm})$ & diff. length $(\mathrm{nm})$ & $S$ par. & $W$ par. & Description \\
\hline Layer 1 & $0-d_{1}$ & 50 & 0.468 & 0.078 & $\mathrm{MgO}$ top layer \\
Layer 2 & $d_{1}-d_{2}$ & 15 & fitted & fitted & $\mathrm{MgO}: \mathrm{Li}$ \\
Layer 3 & $d_{2}-300$ & 50 & fitted & fitted & ion impl. tail \\
Layer 4 & $>300$ & 100 & 0.468 & 0.078 & $\mathrm{MgO}$ bulk \\
\hline \hline
\end{tabular}

${ }^{\mathrm{a}}$ In the case of the $1200 \mathrm{~K}$ annealing step, layer 1 was omitted $\left(d_{1}=0\right)$. 


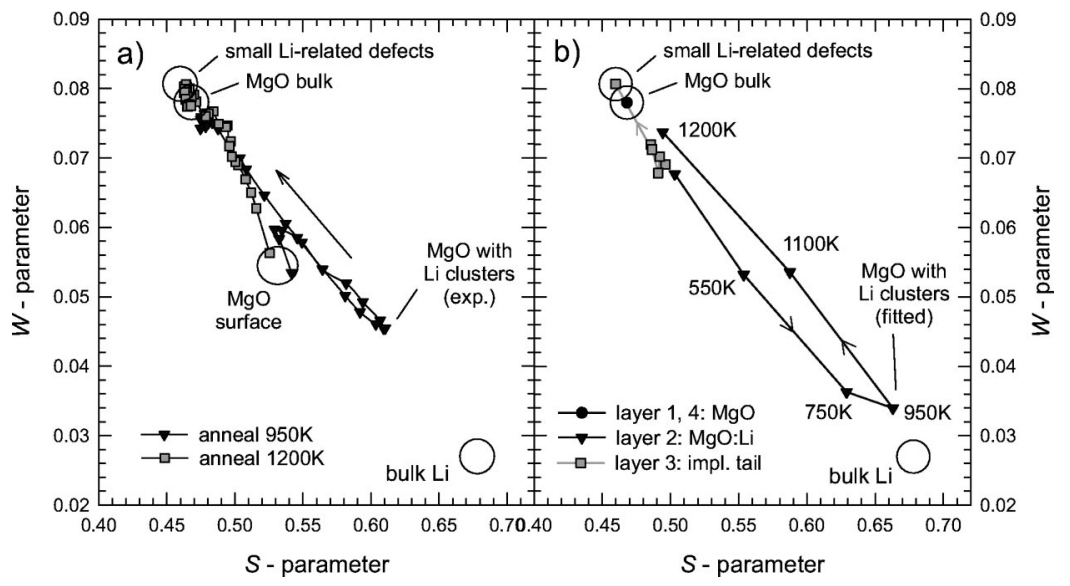

FIG. 4. (a) $S-W$ plot with the positron energy as running parameter. The experimental data are plotted for the $950 \mathrm{~K}$ and $1200 \mathrm{~K}$ annealing step only. (b) $S-W$ plot with the annealing treatment as running parameter. The fitted values are plotted for all of the four layers (see Table II). bulk materials. In Fig. 4(a), the experimentally obtained $S, W$ parameters are plotted with the positron energy as the running parameter. Figure 4(b) shows the $S$-W plot using the fitted $S$ and $W$ values for every layer with the annealing treatment as the running parameter. The main difference between the two plots is that in Fig. 4(a), all layers contribute to the experimental $S, W$ points, while in Fig. 4(b) the $S, W$ parameters are layer resolved with the aid of the VEPFIT code i.e., the $S$ and $W$ parameters corresponding to only one layer are shown [see also Eq. (2)].

Considering the defect evolution of layer 3 (ion implantation tail) in Fig. 4(b), it is clear that the $S, W$ combination moves away from the bulk Li cluster point during the annealing procedure, while the NDP measurements show that a small fraction (approximately 6\%) of the implanted Li atoms is present in this layer. Therefore, the Li atoms should be in an electronic state that is different from metallic bulk Li. Research performed previously on Li-doped $\mathrm{MgO}$ crystals has revealed defect types such as $\mathrm{Li}_{2} \mathrm{O}$ precipitates, isolated $\mathrm{Li}^{+}$ions, and so-called $[\mathrm{Li}]^{0}$ centers. ${ }^{17}$ These defects will yield $S, W$ parameters that are quite different from bulk $\mathrm{Li}$ or bulk $\mathrm{MgO}$. Another observation that can be made with respect to Fig. 4(b) is that the $S, W$ characteristic points corresponding to the ion implantation layer (layer 2) move roughly along the line connecting the $\mathrm{MgO}$ bulk cluster point and the bcc bulk Li cluster point. It is also clear that the experimental $S, W$ point corresponding to $\mathrm{MgO}$ containing $\mathrm{Li}$ clusters after the $950 \mathrm{~K}$ annealing step in Fig. 4(a) does not reach the fitted $S, W$ combination of layer $2(\mathrm{MgO}$ containing lithium clusters) after the $950 \mathrm{~K}$ annealing step in Fig. 4(b). Due to the broadness of the positron implantation profile and positron diffusion processes, only a certain fraction of the positrons implanted at $3.5 \mathrm{keV}$ (with a mean implantation depth that corresponds to the center of the implantation layer) annihilate in the $\mathrm{MgO}: \mathrm{Li}$ layer. This is also clear from Fig. 5 where the fraction of annihilations per layer is plotted as a function of positron implantation energy. It is found that $69 \%$ of the positrons with $3.5 \mathrm{keV}$ implantation energy annihilate in $\mathrm{MgO}: \mathrm{Li}$ (layer 2).

Calculation of the efficiency of positron confinement in $\mathrm{Li}$ nanoclusters using Eq. (1) requires numerical values for the $S$ parameter of the composite $\mathrm{MgO}: \mathrm{Li}$ material (i.e., the $S$ parameter of layer 2), the $S$ parameter of bulk lithium, and the $S$ parameter of bulk $\mathrm{MgO}$. The $S$ parameter of the
MgO:Li layer $\left(S_{\mathrm{MgO}: \mathrm{Li}}\right)$ after the $950 \mathrm{~K}$ annealing step was found to be 0.662 by means of VEPFIT modeling [Table II, Fig. 4(b)]. The $S$ parameters of bulk lithium and bulk $\mathrm{MgO}$ were measured using the same Doppler broadening PBA setup and were evaluated to be 0.678 for polycrystalline bulk bcc Li and 0.468 for monocrystalline bulk MgO. The measurement of the $S$ parameter of bulk Li was carried out at a positron implantation energy of $27 \mathrm{keV}$. This energy is sufficiently high to make the contribution of the lithium oxide layer at the surface to the measured distribution negligible, which was ascertained by means of positron depth profiling. The $S$ parameter for bulk lithium is much higher than the $S$ parameter for bulk $\mathrm{MgO}$. This is due to the rather small Fermi cutoff for metallic lithium of 0.58 a.u. (Ref. 42) (equivalent to $4.2 \mathrm{mrad}$ ) while the window for the measurements of the $S$ parameter is set at energies corresponding to $\pm 3.2 \mathrm{mrad}$. Consequently, most of the Doppler broadening curve falls within the window and the $S$ parameter for lithium is therefore relatively high. This is also clear from Fig. 6 where the experimentally obtained $511 \mathrm{keV}$ annihilation peaks of bulk $\mathrm{MgO}$, bulk bcc $\mathrm{Li}$, and $\mathrm{MgO}: \mathrm{Li}$ are dis-

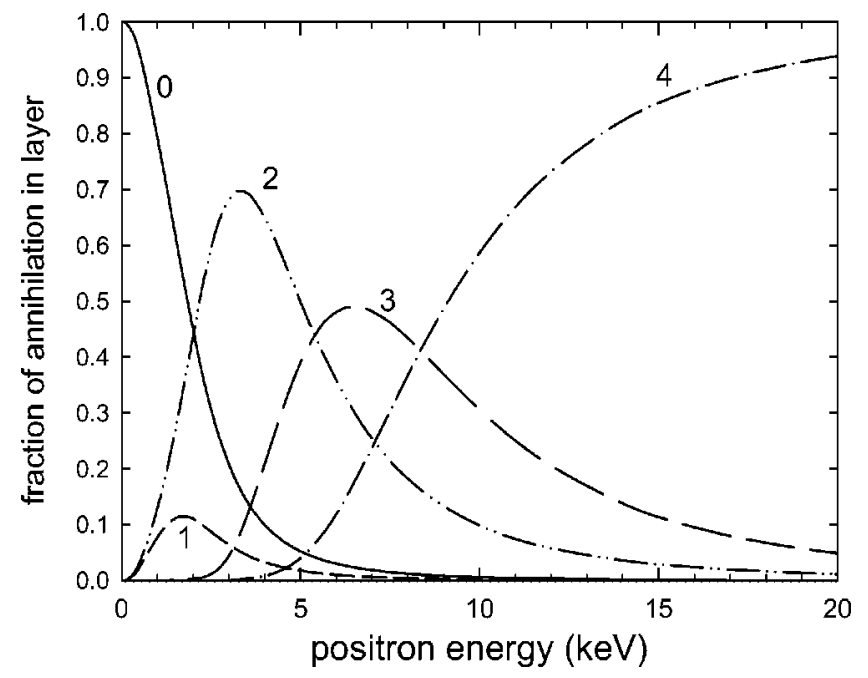

FIG. 5. Layer fractions of the PBA sample as a function of the positron implantation energy. Annihilation fractions per layer were found by VEPFIT modeling of the PBA data obtained after the $950 \mathrm{~K}$ annealing step. The numbers indicate the layer number as listed in Table II. Layer 0 is the surface (thermal and epithermal positrons). 


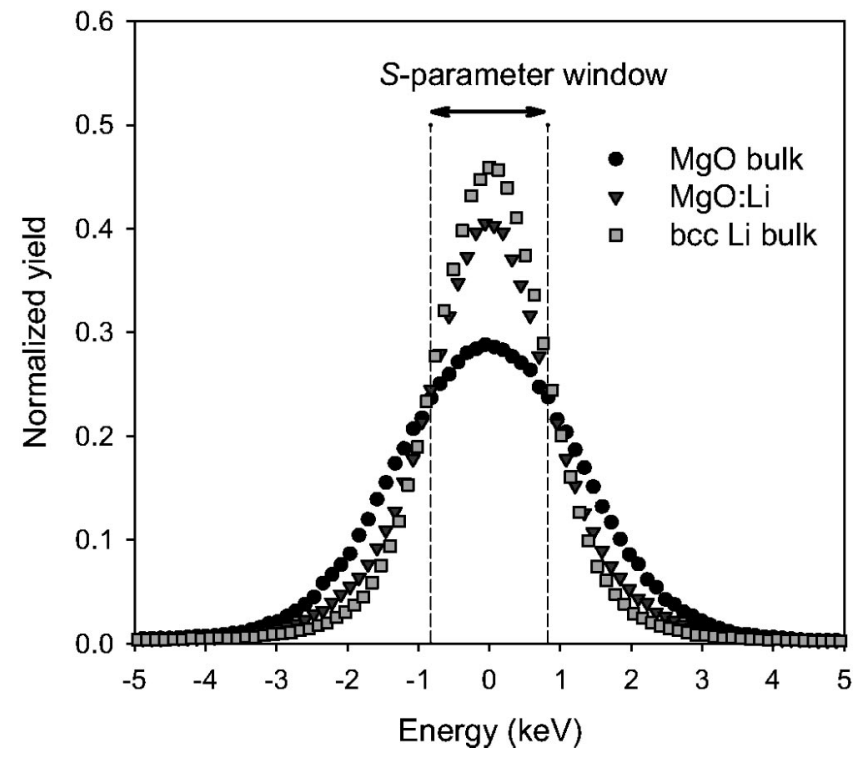

FIG. 6. Experimentally obtained $511 \mathrm{keV}$ Doppler broadening peaks for bulk $\mathrm{MgO}$, bulk bcc $\mathrm{Li}$, and $\mathrm{MgO}$ containing $\mathrm{Li}$ nanoclusters (after the $950 \mathrm{~K}$ annealing step).

played. The fraction of annihilations in lithium can be calculated rewriting Eq. (1) and using $f_{\mathrm{MgO}}+f_{\mathrm{Li}}=1$ :

$$
f_{\mathrm{Li}}=\frac{S_{\mathrm{MgO}: \mathrm{Li}}-S_{\mathrm{MgO}}}{S_{\mathrm{Li}}-S_{\mathrm{MgO}}}
$$

Here it should be realized that contributions from the surface and any other layers have already been filtered out by the VEPFIT code. Substituting the $S$ parameters mentioned above then yields a fraction of annihilations in lithium, $f_{\mathrm{Li}}$, of 92 $\pm 5 \%$. This shows a very effective confinement of positrons in $\mathrm{Li}$ nanoclusters, which occupy only 1.3 at. \% of the $\mathrm{MgO}: \mathrm{Li}$ layer. In the calculation above, it is assumed that the $S$ parameters for bcc Li and fcc Li do not differ significantly and that the $S$ parameter of the lithium nanoclusters (without surrounding $\mathrm{MgO}$ ) is similar to the $S$ parameter of lithium bulk.
A question that now arises is whether the positron is really confined within the Li nanocluster or whether it is trapped at the $\mathrm{MgO} \| \mathrm{Li}$ interface prior to annihilation. We shall postpone the discussion of this point until Sec. V.

\section{B. 2D-ACAR}

The 2D-ACAR results are shown in Fig. 7. Part (a) shows the anisotropic contribution ${ }^{43}$ of the $\mathrm{MgO}$ bulk distribution (total number of counts $6.4 \times 10^{7}$ ) obtained from an asreceived single crystal of $\mathrm{MgO}(100)$. Part (b) of Fig. 7 shows the anisotropic part of the 2D-ACAR distribution obtained from a Li-implanted sample after annealing at $950 \mathrm{~K}$ (total number of counts $\left.1.0 \times 10^{7}\right)$. This distribution was collected at $4 \mathrm{keV}$ positron implantation energy, which corresponds to a mean positron implantation depth at the center of the lithium nanocluster layer ( $\mathrm{MgO}: \mathrm{Li}$ material). This energy was chosen on the basis of depth profiling performed on the 2D-ACAR sample by means of Doppler broadening PBA. Both distributions have been corrected for the difference in resolution between the directions parallel and perpendicular to the sample surface $\left(1.1 \times 1.4 \mathrm{mrad}^{2}\right)$ and were symmetrized by reflection with respect to the (100) and (001) mirror planes. The anisotropy plot of the $\mathrm{MgO}: \mathrm{Li}$ layer is strikingly different from the bulk $\mathrm{MgO}$ anisotropy plot. The bulk $\mathrm{MgO}$ anisotropy is still observable in Fig. 7(b), but another, very dominant anisotropic contribution is present in the central part of the figure. The major part of this contribution has a nearly perfect fourfold symmetry with peaks positioned near the Fermi radius of lithium (see below). Therefore, we attribute the 2D-ACAR anisotropy in the center of Fig. 7(b) to annihilation in bcc or fcc lithium clusters. Treilleux and Chassagne $^{16}$ found by means of electron diffraction and cross-sectional transmission electron microscopy (X-TEM) analysis that, depending on the nanocluster size, metallic lithium nanoclusters in $\mathrm{MgO}$ can adopt either the fcc or a rotated bcc crystal structure. Whether the lithium clusters found in the 2D-ACAR distribution of Fig. 7(b) are in the fcc or rotated bcc phase has not yet been established unequivocally. The fact that the anisotropic contributions displayed in Figs. 7(a) and 7(b) both have the same symmetry and iden-

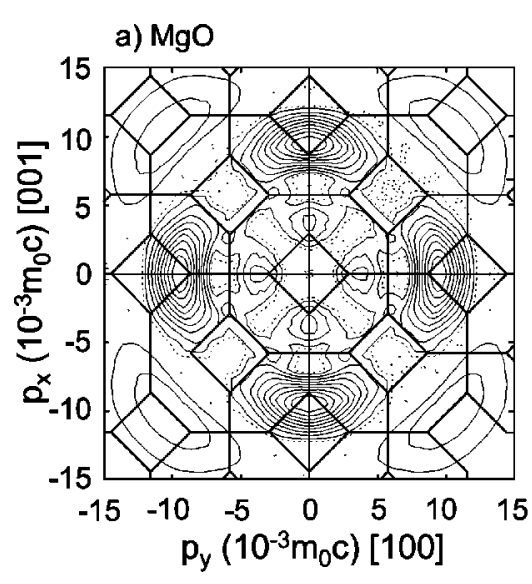

FIG. 7. Anisotropic part of the [010]-axisprojected momentum distribution obtained by 2D-ACAR measurements for (a) bulk $\mathrm{MgO}(100)$ and (b) $\mathrm{MgO}$ containing Li crystalline nanoclusters. The average Fermi cutoff for bulk Li of 4.3 mrad (see Sec. IV. B) is displayed as a dashed circle. The distributions have been symmetrized by reflection with respect to the (100) and (001) mirror planes. The contour interval is $10 \%(5 \%)$ of the peak height for solid (dotted) contours. The projection of the Brillouin zones is shown based on the lattice parameters of $\mathrm{MgO}$ (a) and fcc $\mathrm{Li}$ (b). 
TABLE III. Calculated positron lifetime, positron affinity and electron and positron chemical potentials for bcc and fcc Li.

\begin{tabular}{lccccccc}
\hline \hline & \multicolumn{3}{c}{ LDA } & \multicolumn{2}{c}{ GGA } \\
& $\mu_{-}(\mathrm{eV})$ & $\tau(\mathrm{ps})$ & $\mu_{+}(\mathrm{eV})$ & $A_{+}(\mathrm{eV})$ & $\tau(\mathrm{ps})$ & $\mu_{+}(\mathrm{eV})$ & $A_{+}(\mathrm{eV})$ \\
\hline bcc Li & -2.8 & 299 & -5.2 & -8.0 & 283 & -5.0 & -7.8 \\
fcc Li & -2.8 & 297 & -5.3 & -8.1 & 282 & -5.0 & -7.8 \\
\hline \hline
\end{tabular}

tical mirror planes shows that there is a distinct orientation relationship between the crystalline Li clusters and the $\mathrm{MgO}$ host matrix. Therefore, the lithium clusters will be coherent or semicoherent with the $\mathrm{MgO}$ crystal lattice. Comparing the ion implantation settings and annealing temperature used in this work with the experimental parameters of Ref. 16, the presence of fcc lithium is most likely. In that case, the lithium nanoclusters are in a simple cube-on-cube orientation relationship with the $\mathrm{MgO}$ host matrix. Cube-on-cube bcc Li is excluded by the anisotropy in Fig. 7(b) as it would produce peaks along the $\langle 100\rangle$ directions. The anisotropy in the central part of Fig. 7(b) agrees very well with the results of preliminary calculations performed on the momentum distribution of fcc Li using the Korringa-Kohn-Rostoker (KKR) method. The free-electron Fermi sphere for fcc Li $\left(a_{0}\right.$ $=4.4 \AA$; cf. Table I) is displayed in Fig. 7(b) as a circle of radius 0.591 a.u., equivalent to $4.3 \mathrm{mrad}$. The peaks in the anisotropy then derive from bulges of the Fermi surface pointing towards the hexagonal Brillouin zone faces. The results of the momentum density calculations for fcc and bcc $\mathrm{Li}$ and a more detailed analysis of the experimental 2DACAR distributions will be presented elsewhere. ${ }^{44}$

The fraction of positron annihilations in Li nanoclusters $f_{\mathrm{Li}}$ was also estimated from the 2D-ACAR distribution, using Eq. (1) in combination with a VEPFIT layer fraction analysis (giving the relative contributions of the layers to the annihilation distribution at a certain positron implantation energy). The fraction found $(90 \%)$ was not significantly different from the one derived from the PBA results discussed in Sec. IV A.

\section{Positron affinities}

Above it was found that the fraction of annihilations in $\mathrm{Li}$ as deduced from the Doppler broadening PBA spectra is 92 $\pm 5 \%$ in the ion-implanted layer. It will be shown that the effective positron confinement can be ascribed to the differ- ent positron affinities of $\mathrm{MgO}$ and $\mathrm{Li}$. These positron affinities were calculated using the various models discussed in Sec. II. The results of calculations of the positron lifetime, affinity, and electron and positron chemical potentials for $\mathrm{Li}$ are shown in Table III. Very similar lifetimes and affinities are obtained for bcc and fcc Li. This is related to the fact that the atomic volumes of bcc and fcc Li are almost equal (Table I). The affinities found are in good agreement with theoretical values from the literature. ${ }^{45,26}$ To our knowledge, the positron affinity of Li has never been determined experimentally.

The electron and positron chemical potentials, positron affinity and lifetime for $\mathrm{MgO}$ determined using three different models are given in Table IV. The IM and GGA approaches yield values for the bulk lifetime that are close to the experimentally obtained lifetime $\tau$ of $150-170$ ps in $\mathrm{MgO},{ }^{46,47}$ which is not the case for the SM results. We note that the incorporation of empty spheres into the $\mathrm{MgO}$ lattice ( $\mathrm{MgO}$ II case) leads to a slight increase of the lifetime values because the interstitial space is better described compared to the case without empty spheres (MgO I). Table IV also includes the calculated lifetimes by Puska et al. ${ }^{35}$ In the present work, the lifetimes found using the SM and the IM are somewhat higher and lower, respectively, than the values reported by Puska et al., while the same SM and IM models have been used. This is mainly due to a different treatment of the core electrons. In addition, the electron density of $\mathrm{MgO}$ in Ref. 26 was approximated by the superposition of atomic densities without consideration of the charge transfer between $\mathrm{Mg}$ and $\mathrm{O}$ atoms. Anyhow, it turns out that the semiconductor model does not describe reasonably the lifetime experiment (both in the present work and in Ref. 26) so that we will not consider this model when calculating the difference between positron affinities of $\mathrm{MgO}$ and $\mathrm{Li}$.

The values of $A_{+}$presented in Table IV depend obviously on the models used to calculate them. The lowest value

TABLE IV. Calculated lifetimes, positron affinities, and electron and positron chemical potentials for $\mathrm{MgO}$ without empty spheres ( $\mathrm{MgO}$ I) and including empty spheres ( $\mathrm{MgO}$ II). GGA: generalized gradient approximation. SM: semiconductor model. IM: insulator model.

\begin{tabular}{lcccccccccc}
\hline \hline & & \multicolumn{3}{c}{ GGA } & \multicolumn{3}{c}{ SM } & & IM & \\
& $\mu_{-}$ & $\tau$ & $\mu_{+}$ & $A_{+}$ & $\tau$ & $\mu_{+}$ & $A_{+}$ & $\tau$ & $\mu_{+}$ & $A_{+}$ \\
& $(\mathrm{eV})$ & $(\mathrm{ps})$ & $(\mathrm{eV})$ & $(\mathrm{eV})$ & $(\mathrm{ps})$ & $(\mathrm{eV})$ & $(\mathrm{eV})$ & $(\mathrm{ps})$ & $(\mathrm{eV})$ & $(\mathrm{eV})$ \\
\hline $\mathrm{MgO} \mathrm{I}$ & -2.9 & 139 & -3.2 & -6.1 & 121 & -4.0 & -6.9 & 141 & -2.6 & -5.5 \\
$\mathrm{MgO}^{\mathrm{a}}$ & -1.0 & 140 & -5.0 & -6.0 & 126 & -5.5 & -6.5 & 147 & -4.0 & -5.0 \\
$\mathrm{MgO}^{\mathrm{a}}$ & & & & & 119 & & & 167 & & \\
\hline \hline
\end{tabular}

${ }^{\mathrm{a}}$ Ref. 35. 
comes from the SM, whereas the IM yields the largest value. Also, the affinity results depend slightly on whether the empty spheres are included or not. In the following considerations we will employ the $\mathrm{MgO}$ II case. The positronic energy step at the $\mathrm{MgO} \| \mathrm{Li}$ interface can now be calculated using Eq. (4). Combining the GGA and IM positron affinities for $\mathrm{MgO}$ II (Table IV) and the GGA results for bcc and fcc lithium (Table III; LDA and GGA results differ only marginally), the positronic potential drop at the $\mathrm{MgO} \| \mathrm{Li}$ interface equals $1.8 \mathrm{eV}$ using the GGA and $2.8 \mathrm{eV}$ using the IM. Regardless of this uncertainty in the affinity difference, it is plausible to assume that clusters of $\mathrm{Li}$ in $\mathrm{MgO}$ act as positron traps.

In this context it is useful to mention recent work $^{38}$ in which the positron affinity was studied in several polytypes of $\mathrm{SiC}$, which is a semiconductor. It was found that all theoretical approaches (GGA, SM, IM) overestimate (in magnitude) the positron affinity in SiC. This was ascribed to certain inadequacies in the theoretical description of both the electron and the positron part of the problem. Considering this conclusion and the above mentioned experimental values of the positron affinity for $\mathrm{MgO}$ (Refs. 29 and 30) it turns out that the values of $A_{+}$calculated here for $\mathrm{MgO}$ are probably too large (in magnitude). Further theoretical and experimental work is therefore needed to clarify this point.

A final remark in this section concerns the sensitivity of the values of the positron affinity with respect to the choice of the LDA exchange-correlation potential for electrons. In addition to the exchange-correlation potential of Von Barth and $\operatorname{Hedin}^{37}$ we also examined the potential of Ceperley and Alder $^{48}$ as parametrized by Perdew and Zunger. ${ }^{49}$ Surprisingly, the calculated positron affinities increase (magnitudes decrease) by about $0.5 \mathrm{eV}$ in all cases presented in Tables III and IV. The inspection of electron and positron chemical potentials leads to the conclusion that this effect can be attributed to the change of the electron chemical potentials only. It shows that, although the resulting difference of the positron affinity of $\mathrm{MgO}$ and $\mathrm{Li}$ remains unchanged, one has to be careful when comparing affinity results obtained using different exchange-correlation potentials for electrons (cf. Ref. 50).

\section{DISCUSSION}

Our results show a very high fraction of annihilations in lithium, indicating a very effective positron confinement in Li nanoclusters. Two aspects are important when considering the efficiency of positron confinement in nanoclusters: (a) the probability of trapping and reflection when a positron encounters a nanocluster acting as a potential well and (b) the probability of reaching a nanocluster, assuming that the positron is thermalized in the embedding $\mathrm{MgO}$ material.

The kinetic energy of delocalized positrons is of the order of tens of $\mathrm{meV}$, while the size of the potential step at the $\mathrm{MgO} \| \mathrm{Li}$ interface is a few $\mathrm{eV}(1.8 \mathrm{eV}$ using the GGA and 2.8 $\mathrm{eV}$ using the insulator model). Thus, the depth of the potential well as determined by the difference in positron affinities is two orders of magnitude larger than the kinetic energy of the positron after thermalization. Therefore, when a positron is thermalized and diffuses within the embedding $\mathrm{MgO}$, the probability of reflection when encountering the $\mathrm{MgO} \| \mathrm{Li}$ interface is negligible at room temperature. Every positron reaching a lithium nanocluster will thus be confined in that cluster. Once inside the cluster, the positron will be reflected at the walls. A necessary condition for the process described above is that the size of the nanoclusters is large enough to accommodate at least one bound state. Applying the threedimensional spherical well model with a finite potential, the critical radius is given by Eq. (5). Substituting a value of 1.8 $\mathrm{eV}$ for the depth of the potential well then yields a critical cluster diameter of $0.4 \mathrm{~nm}$. As the confinement of positrons is quite obvious from the results described in Secs. IV A and IV B, the actual size of the nanoclusters will be larger than this value.

The probability of encountering a nanocluster is determined by the positron diffusion length and the concentration and spatial distribution of the Li clusters. Trapping of the vast majority of the positrons is only possible if the mean distance between the Li nanoclusters is smaller than the diffusion length for positrons diffusing in $\mathrm{MgO}$ within the $\mathrm{MgO}: \mathrm{Li}$ layer. Here it is assumed that the positron is present in $\mathrm{MgO}$ after thermalization, as Li occupies only 3 vol. \% of the MgO:Li layer. In the diffusion-limited trapping model, described by Hautojärvi and Corbel, ${ }^{51}$ the diffusion to the defect is slow compared to the transition to the localized state. The depth of the nanocluster potential well is of the order of a few $\mathrm{eV}$, so this condition is well satisfied. The possibility of detrapping is neglected. Assuming spherically shaped clusters, the fraction of positrons annihilating in the clusters is given by

$$
f_{\mathrm{Li}}=\frac{\kappa}{\kappa+\lambda_{\mathrm{MgO}}}=\frac{4 \pi r_{c l} D_{+} c_{c l}}{4 \pi r_{c l} D_{+} c_{c l}+\lambda_{\mathrm{MgO}}},
$$

where $\kappa$ is the positron trapping rate with respect to the clusters $\left(\mathrm{s}^{-1}\right), \lambda_{\mathrm{MgO}}$ is the annihilation rate in $\mathrm{MgO}\left(\mathrm{s}^{-1}\right)$, $r_{c l}$ is the radius of the cluster (m), $c_{c l}$ is the concentration of clusters $\left(\mathrm{m}^{-3}\right)$, and $D_{+}$is the diffusion coefficient $\left(\mathrm{m}^{2} \mathrm{~s}^{-1}\right)$. The diffusion coefficient is related to the diffusion length $L_{+}$ (m) and to the positron lifetime $\tau(\mathrm{s})$ as

$$
L_{+}=\sqrt{D_{+} \tau} \text {. }
$$

By means of Eqs. (9) and (10), the fraction of annihilations in lithium clusters can be estimated. No detailed information is available on the size of the nanoclusters, so we perform an order-of-magnitude calculation. The mass density of bulk Li is $543 \mathrm{~kg} \mathrm{~m}^{-3}$, so the $10^{16}$ implanted $\mathrm{Li}$ atoms occupy a total volume of $2.1 \times 10^{-13} \mathrm{~m}^{3}$ independent of the cluster size. If the radius of the lithium nanoclusters is, e.g., 5.0 $\mathrm{nm},{ }^{16}$ the total number of clusters is $4.1 \times 10^{11}$ and the concentration of clusters will be $5.8 \times 10^{22} \mathrm{~m}^{-3}$ considering that all clusters are present in the ion implantation layer with a thickness of $70 \mathrm{~nm}$ (estimated from VEPFIT, Table II) and an area of $1.0 \times 1.0 \mathrm{~cm}^{2}$. At this concentration, the average intercluster distance is $26 \mathrm{~nm}$. The annihilation rate in $\mathrm{MgO}$, $\lambda_{\mathrm{MgO}}$, equals $6.5 \times 10^{9} \mathrm{~s}^{-1}$ as it is the inverse of the lifetime $\tau$ in $\mathrm{MgO}, 155 \mathrm{ps.}^{46} \mathrm{~A}$ value for the diffusion length for positrons in $\mathrm{MgO}$ of $50 \mathrm{~nm}$ can be assumed (equal to the 
diffusion lengths for layers 1 and 3 in the VEPFIT model). Substituting Eq. (10) into Eq. (9) and using the numerical values given above, the fraction of annihilations in $\mathrm{Li}, f_{\mathrm{Li}}$, can be calculated. This yields a fraction of $90 \%$, which agrees very well with the experimental results. Although the cluster size and the diffusion length are estimated, the above calculation demonstrates that the annihilation fraction in lithium can be lower than $100 \%$ due to diffusion-related processes even if every positron encountering a nanocluster is trapped in that cluster. The calculated fraction is strongly dependent on the cluster size and the diffusion length. For clusters with a radius of 3-7 nm and a diffusion length of $50-100 \mathrm{~nm}$, the trapped fraction varies from $82 \%$ to $99 \%$.

Finally, the possibility should be discussed that positrons are not present in either $\mathrm{MgO}$ or $\mathrm{Li}$ but are trapped at the interface, corresponding to the situation depicted in Fig. 2(c). This phenomenon is observed, e.g., for the $\mathrm{Si} \| \mathrm{SiO}_{2}$ interface. ${ }^{27}$ Open volume defects or lattice mismatch at the interface can induce positron trapping. No evidence of positronium formation was found in the analysis of the 2DACAR distribution, showing that large vacancy-type defects are not present. Positrons can also trap in interface defects that are too small for the formation of positronium. However, positron annihilation distributions corresponding to positron annihilation at interfaces usually bear characteristics of both materials. Thus, if positron trapping would occur at the $\mathrm{MgO} \| \mathrm{Li}$ interface, a considerably higher contribution from $\mathrm{MgO}$ to the Doppler broadening and 2D-ACAR distributions would be expected. Therefore, interface trapping in the case of the $\mathrm{MgO} \| \mathrm{Li}$ interface is not likely on the basis of the above observations. On the other hand, the fraction of annihilations in $\mathrm{Li}$ nanoclusters might be influenced by local electric fields near the interface. These can be present as a result of charge redistribution associated with the equalization of the Fermi levels of $\mathrm{MgO}$ and $\mathrm{Li}$ in thermal equilibrium. We have, however, no means to investigate whether this last aspect plays a significant role.

\section{CONCLUSIONS}

It has been shown that positrons are very effectively confined in crystalline lithium nanoclusters that are embedded in $\mathrm{MgO}$. The fraction of positron annihilations in lithium nanoclusters was deduced by analysis of the Doppler broadening PBA spectra and found to be $92 \pm 5 \%$. This annihilation fraction is very high, considering the fact that the lithium content in the MgO:Li layer is only 1.3 at. \%. The anisotropic contribution of the 2D-ACAR distribution collected in the layer containing Li nanoclusters shows a fourfold symmetry (most likely indicating an fcc crystal structure) with peaks at positions near the average Fermi radius of lithium. This proves that crystalline bulk lithium is present. The symmetry of the 2D-ACAR anisotropic contributions also shows that the lithium clusters are coherent or semicoherent with the $\mathrm{MgO}$ host matrix. The difference in positron affinities of lithium and $\mathrm{MgO}$ yields a positronic potential step down at the $\mathrm{MgO} \| \mathrm{Li}$ interface. Therefore, the nanoclusters act as a potential well for positrons with a depth equal to the difference in the positron affinities. These positron affinities were calculated using the LMTO-ASA method, and values for the depth of the positronic potential well of 1.8 and $2.8 \mathrm{eV}$ were obtained using the GGA and the insulator model, respectively. The depth of the potential well is so large that the trapping probability of positrons meeting a nanocluster is approximately equal to 1 .

In future research, positron confinement can facilitate the investigation of metal and semiconductor quantum dots and of unusual crystal structures, such as the electronic structure of fcc $\mathrm{Li}$ nanoclusters in $\mathrm{MgO}$ or of bcc $\mathrm{Cu}$ nanoclusters in $\mathrm{Fe}$. In order to predict beforehand whether positron confinement is feasible, it would be useful to know the experimentally determined or calculated positron affinities of embedding transparent materials commonly used in optical studies, such as $\mathrm{SiO}_{2}, \mathrm{TiO}_{2}$, and $\mathrm{Al}_{2} \mathrm{O}_{3}$. These affinities can then be compared with the known positron affinities of many nanocluster materials.

Note added in proof. Very recently, Nagai et al. have investigated successfully the Fermi surface of coherently embedded $\mathrm{Cu}$ nanoclusters having the unusual bcc crystal structure. ${ }^{53}$ Here the concept of position confinement in embedded nanoclusters was used in combination with the $2 \mathrm{D}$ ACAR technique.

\section{ACKNOWLEDGMENTS}

Thanks are due to O.K. Andersen and O. Jepsen for providing their LMTO-ASA code. We are indebted to M.J. Puska and T. Korhonen for permitting the use of their LMTO positron code and for stimulating discussions. We acknowledge Y. Nagai for valuable discussions on the subject of positron confinement. Thanks are also due to F. Labohm for the NDP measurements and a discussion of the results.
${ }^{1}$ P. Chakraborty, J. Mater. Sci. 33, 2235 (1998).

${ }^{2}$ U. Kreibig and M. Vollmer, Optical Properties of Metal Clusters, Springer Series in Materials Science, Vol. 25 (Springer, Berlin, 1995).

${ }^{3}$ K. Fukumi, A. Chayahara, K. Kodano, T. Sakaguchi, Y. Horino, M. Miya, K. Jujii, J. Hayakawa, and M. Satou, J. Appl. Phys. 75, 3075 (1994).

${ }^{4}$ Metal Clusters, edited by W. Ekardt (Wiley, New York, 1999).

${ }^{5}$ Semiconductor Nanoclusters, edited by P.V. Kamat and D. Meisel
(Elsevier, New York, 1997).

${ }^{6}$ D. Davidovic and M. Tinkham, Phys. Rev. Lett. 83, 1644 (1999).

${ }^{7}$ A.P. Alivisatos, Science 271, 933 (1996).

${ }^{8}$ Positron Spectroscopy of Solids, edited by A. Dupasquier and A.P. Mills, Jr. (IOS Press, Amsterdam, 1995).

${ }^{9}$ M.J. Puska and R.M. Nieminen, Rev. Mod. Phys. 66, 841 (1994).

${ }^{10}$ R. N. West, in Positron Spectroscopy of Solids (Ref. 8), p. 75.

${ }^{11}$ S.S. Rajput, R. Prasad, R.M. Singru, W. Trifthäuser, A. Eckert, G. Kögel, S. Kaprzyk, and A. Bansil, J. Phys.: Condens. Matter 5, 
6419 (1993).

${ }^{12}$ Y. Nagai, M. Hasegawa, Z. Tang, A. Hempel, K. Yubuta, T. Shimamura, Y. Kawazoe, A. Kawai, and F. Kano, Phys. Rev. B 61, 6574 (2000).

${ }^{13}$ C.W. White, J.D. Budai, S.P. Withrow, J.G. Zhu, E. Sonder, R.A. Zuhr, A. Meldrum, D.M. Hembree, Jr., D.O. Henderson, and S. Prawer, Nucl. Instrum. Methods Phys. Res. B 141, 228 (1998).

${ }^{14}$ A. Vom Felde, J. Fink, and W. Ekardt, Phys. Rev. Lett. 61, 2249 (1988).

${ }^{15}$ G. Marichy, G. Chassagne, and D. Durand, Phys. Status Solidi B 92, 221 (1979).

${ }^{16}$ M. Treilleux and G. Chassagne, J. Phys. (Paris) 40, L-283 (1979); J. Phys. (Paris), Colloq. 41, C6-391 (1980).

${ }^{17}$ I. Vergara, R. González, R. Ramírez, J.E. Muñoz Santiuste, and Y. Chen, Phys. Rev. B 58, 5236 (1998).

${ }^{18}$ N.E. Christensen and D.L. Novikov, Phys. Rev. Lett. 86, 1861 (2001).

${ }^{19}$ V.G. Vaks, M.I. Katsnelson, V.G. Koreshkov, A.I. Likhtenstein, O.E. Parfenov, V.F. Skok, V.A. Sukhoparov, A.V. Trefilov, and A.A. Chernyshov, J. Phys.: Condens. Matter 1, 5319 (1989).

${ }^{20}$ A. van Veen, M.A. van Huis, A.V. Fedorov, H. Schut, C.V. Falub, S.W.H. Eijt, F. Labohm, B.J. Kooi, and J.Th.M. De Hosson, in Ion Beam Synthesis and Processing of Advanced Materials, edited by S. C. Moss, K.-H. Heining, and D. B. Poker, Mater. Res. Soc. Symp. Proc. No. 647 (Materials Research Society, Pittsburgh 2001), p. O11.24.

${ }^{21}$ A. van Veen, H. Schut, and P. E. Mijnarends, in Positron Beams and their Applications, edited by P. Coleman (World Scientific, Singapore, 2000), p. 191.

${ }^{22}$ M. Clement, J.M.M. de Nijs, P. Balk, H. Schut, and A. van Veen, J. Appl. Phys. 79, 9029 (1996).

${ }^{23}$ A. van Veen, H. Schut, J. de Vries, R.A. Hakvoort, and M.R. IJpma, in Positron Beams for Solids and Surfaces, AIP Conf. Proc. 218, edited by P. J. Schultz, G. R. Massoumi, and P. J. Simpson (AIP, New York, 1990), p. 171.

${ }^{24}$ A.V. Fedorov, A. van Veen, and H. Schut, Mater. Sci. Forum 363-365, 646 (2001).

${ }^{25}$ A. Ueda, R. Mu, Y.-S. Tung, M. Wu, W.E. Collins, D.O. Henderson, C.W. White, R.A. Zuhr, J.D. Budai, A. Meldrum, P.W. Wang, and Xi Li, Nucl. Instrum. Methods Phys. Res. B 141, 261 (1998)

${ }^{26}$ M.J. Puska, P. Lanki, and R.M. Nieminen, J. Phys.: Condens. Matter 1, 6081 (1989).

${ }^{27}$ P. Asoka-Kumar, K.G. Lynn, and D.O. Welch, J. Appl. Phys. 76, 4935 (1994).

${ }^{28}$ A. H. Weiss and P. G. Coleman, in Positron Beams and their Applications, edited by P. Coleman (World Scientific, Singapore, 2000), p. 129.

${ }^{29}$ P. Sferlazzo, S. Berko, and K.F. Canter, Phys. Rev. B 35, 5315 (1987).

${ }^{30}$ Y. Nagashima, Y. Morinaka, T. Kurihara, Y. Nagai, T. Hyodo, T. Shidara, and K. Nakahara, Phys. Rev. B 58, 12676 (1998).
${ }^{31}$ For a recent review, see O. K. Andersen, O. Jepsen, and M. Šob, in Electronic Band Structure and its Applications, edited by M. Yussouff (Springer, Berlin, 1987), p. 1.

${ }^{32}$ H. L. Skriver, The LMTO Method (Springer, Berlin, 1984).

${ }^{33}$ E. Boroński and R.M. Nieminen, Phys. Rev. B 34, 3820 (1986).

${ }^{34}$ B. Barbiellini, M.J. Puska, T. Torsti, and R.M. Nieminen, Phys. Rev. B 51, 7341 (1995); B. Barbiellini, M.J. Puska, T. Korhonen, A. Harju, T. Torsti, and R.M. Nieminen, ibid. 53, 16201 (1996).

${ }^{35}$ M.J. Puska, S. Mäkinen, M. Manninen, and R.M. Nieminen, Phys. Rev. B 39, 7666 (1989).

${ }^{36}$ D. M. Roessler and D. R. Huffman, in Handbook of Optical Constants of Solids II, edited by E. D. Palik (Academic Press, London, 1991), p. 919.

${ }^{37}$ U. Von Barth and L. Hedin, J. Phys. C 5, 1629 (1972).

${ }^{38}$ J. Kuriplach, M. Šob, G. Brauer, W. Anwand, E.-M. Nicht, P.G. Coleman, and N. Wagner, Phys. Rev. B 59, 1948 (1999).

${ }^{39}$ C.V. Falub, S.W.H. Eijt, A. van Veen, P.E. Mijnarends, and H. Schut, Mater. Sci. Forum 363-365, 561 (2001).

${ }^{40}$ B.J. Kooi, A. van Veen, J.Th.M. De Hosson, H. Schut, A.V. Fedorov, and F. Labohm, Appl. Phys. Lett. 76, 1110 (2000).

${ }^{41}$ M.A. van Huis, A.V. Fedorov, A. van Veen, F. Labohm, H. Schut, P.E. Mijnarends, B.J. Kooi, and J.Th.M. De Hosson, Mater. Sci. Forum 363-365, 448 (2001).

${ }^{42}$ Y. Tanaka, Y. Sakurai, A.T. Stewart, N. Shiotani, P.E. Mijnarends, S. Kaprzyk, and A. Bansil, Phys. Rev. B 63, 045120 (2001).

${ }^{43}$ The anisotropic part of the 2D-ACAR distribution was determined by subtracting an isotropic distribution that everywhere remains within the measured distribution. The anisotropy is therefore everywhere positive.

${ }^{44}$ C. V. Falub, S. W. H. Eijt, M. A. van Huis, A. van Veen, H. Schut, and P. E. Mijnarends, Nucl. Instrum. Meth. Phys. Res. B (to be published); C. V. Falub et al. (unpublished).

${ }^{45}$ M. Farjam and H.B. Shore, Phys. Rev. B 36, 5089 (1987).

${ }^{46}$ M. Forster, J. N. Mundy, and H.-E. Schaefer, in Positron Annihilation, edited by L. Dorikens-Vanpraet, M. Dorikens, and D. Segers (World Scientific, Singapore, 1989), p. 833.

${ }^{47}$ R. Pareja, M.A. Pedrosa, and R. Gonzalez, in Positron Annihilation, edited by P. C. Jain, R. M. Singru, and K. P. Gopinathan (World Scientific, Singapore, 1985), p. 708.

${ }^{48}$ D.M. Ceperley and B.J. Alder, Phys. Rev. Lett. 45, 566 (1980).

${ }^{49}$ J.P. Perdew and A. Zunger, Phys. Rev. B 23, 5048 (1981).

${ }^{50}$ B.K. Panda, G. Brauer, W. Skorupa, and J. Kuriplach, Phys. Rev. B 61, 15848 (2000).

${ }^{51}$ P. Hautojärvi and C. Corbel, in Positron Spectroscopy of Solids (Ref. 8), p. 491.

${ }^{52}$ Handbook of Chemistry and Physics, 56th ed. (CRC Press, Boca Raton, 1975), p. B-214.

${ }^{53}$ Y. Nagai, T. Chiba, Z. Tang, T. Akahane, T. Kanai, M. Hasegawa, M. Takenaka, and E. Kuramoto, Phys. Rev. Lett. 87, 176402 (2001). 